\title{
Medial pantarsal arthrodesis with Compact UniLock 2.0 locking plate system in a cat
}

\author{
Luca Pennasilico, Riccardo Botto, Caterina Di Bella* and Angela Palumbo Piccionello \\ School of Biosciences and Veterinary Medicine, University of Camerino, Camerino, Italy
}

\begin{abstract}
Background: Pantarsal arthrodesis is a salvage procedure carried out for the treatment of tarsal joint disease, including severe osteoarthritis with intractable pain in the tarsocrural joint, tarsal fractures, shearing injuries, tarsocrural joint instability, and failure of the common calcaneal tendon. Although medial plating is preferable, the high incidence of post-surgery complications is possible. Using thin, pre-contouring or easy contouring, locking plates might reduce the incidence of such complications. However, to date, there are no pre-contouring and dedicated locking plates for pantarsal arthrodesis with medial placement.

Case Description: The case of an 18-month-old female stray European cat has been referred because of a severe tibiotarsal injury improperly treated with an intramedullary pin. The patient was submitted for medial pantarsal arthrodesis, performed with the Compact UniLock 2.0 $0^{\mathrm{TM}}$ locking plate systema (DePuy Synthes, Oberdorf, Switzerland). The authors hypothesized that this particularly innovative osteosynthesis system could present advantages compared to the systems already in use for medial pantarsal arthrodesis and therefore reduce the risk of complications.

Conclusion: This innovative titanium locking system, because of its versatile contour function and thinness, allowed the good functional recovery of the limb and showed numerous advantages over traditional systems.

Keywords: Internal fixator, Medial plate, Pantarsal arthrodesis, Tarsal injury, UniLock system.
\end{abstract}

\section{Introduction}

Pantarsal arthrodesis is a challenging procedure and a high incidence of complications has been reported, ranging from $30 \%$ to $70 \%$ (Klause et al., 1989; Doverspike and Vasseur, 1991; Gorse et al., 1991; Roch et al., 2008). The most reliable results are obtained by using bone plates to stabilize the arthrodesis, which can be applied in any aspect of the joint surface (Sumner-Smith and Kuzma, 1989; Dyce et al., 1998; Wilke et al., 2000; MecKe et al., 2004). Although plantar plating is the best biomechanical choice since the plate is positioned on the tension surface, dorsal or medial plating is preferable (Wilke et al., 2000; Spencer and Tobias, 2018). Dorsal plates are applied to the compression aspect of the joint and may be prone to failure because of cyclic loading and implant loosening or plate failure (Anesi et al., 2019). For this reason, medial plating has biomechanical advantages over dorsally positioned plates, but a high incidence of complications has been associated with medially positioned plates (Decamp et al., 1993). Complications reported after pantarsal arthrodesis include implant failure (Decamp et al., 1993; Jaeger et al., 2007), calcaneal or metatarsal fractures, delayed wound healing, surgical site infection, Achilles tendon pain (Anesi et al., 2019), and plantar necrosis (Roch et al., 2008), particularly, even if not completely understood, when the medial part of the joint has been plated. The plate thickness and the marked soft tissue retraction may often cause excessive tension, resulting in surgical wound dehiscence (Fitzpatrick et al., 2013). The absence, to date, of locking precontoured plates, with respect to the functional joint angle (115 $-125^{\circ}$ in cats) (Spencer and Tobias, 2018), and the conspicuous thickness of the implants still limit the therapeutic success of medial plating in cats. The Compact UniLock system was originally designed for human maxillofacial surgery and functions as an internal fixator; the plates are made of titanium, and the screws are titanium alloys and self-tapping (Streubel et al., 2011). The locking mechanism of the Compact UniLock system consists of threaded screw heads which lock in the corresponding threads of the plate (Keller et al., 2005). The locking mechanism and the versatile contour function make this system highly adaptable (Keller et al., 2005; Şirin et al., 2018). Although its use has been reported in a variety of applications in small animal orthopedics, even for the treatment of joint diseases, none of the previous publications has reported its use in pantarsal arthrodesis medial plating for cats. The purpose of this report is to describe the use and outcome of the Compact 2.0 UniLock system in medial tarso-pantarsal arthrodesis in a cat. We hypothesized that the system would be adequate regarding practicability, stability, and overall outcome and that it would present some advantages over currently available systems. 


\section{Case Details}

An 18-month-old female stray European cat was referred to the Veterinary Teaching Hospital of the University of Camerino, Italy. Anamnestic data reported a distal tibial epiphyseal sliding fracture (SH1) of the right hind limb inadequately treated 8 months prior with an intramedullary pin by colleagues and consequent chronic tarsocrural instability. Gait evaluation showed a non-weight bearing lameness (grade 4/5) of the right hind limb. An orthopedic examination revealed severe pain during the manipulation of the right tarsus, with bone crackling during stressed manipulation elicited by tibiotarsal joint laxity. Mediolateral and craniocaudal projections of the right tarsus showed failure of the fracture implant (intramedullary pin) caused by an inadequate choice of the apparatus and an incorrect osteosynthesis technique, with a caudomedial displacement of the right distal tibial epiphysis and a moderate process of bone remodeling and resorption (Fig. 1). The patient underwent pantarsal arthrodesis for the recovery of limb functionality. The anesthetic protocol included premedication with $3 \mu \mathrm{g} / \mathrm{kg}$ of dexmedetomidine (Dexdomitor $0.5 \mathrm{mg} / \mathrm{ml}$; Orion Corporation, Finland), $0.3 \mathrm{mg} / \mathrm{kg}$ of methadone (Semfortan $10 \mathrm{mg} / \mathrm{ml}$; Dechra, Italy), and $3 \mathrm{mg} / \mathrm{kg}$ of ketamine (Ketavet 100; Intervet Productions s.r.l., Italy) administered intramuscularly (IM). General anesthesia was induced with $4 \mathrm{mg} / \mathrm{kg}$ of propofol (Fresenius Kabi, Isola della Scala, Italy) administered intravenously (IV) and maintained with oxygen and isoflurane. When a good anesthesia plan was achieved, the femoral-sciatic

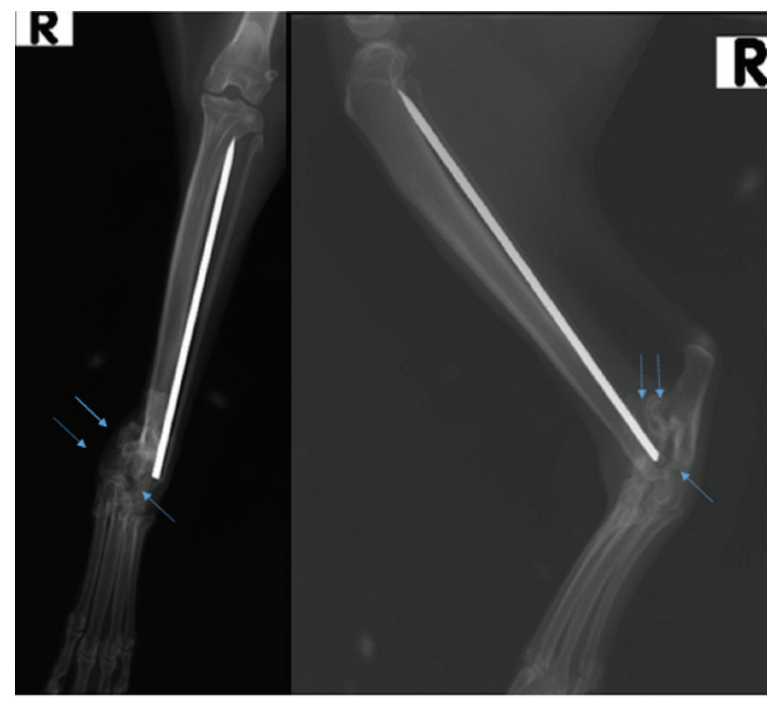

Fig. 1. Craniocaudal and mediolateral radiographs of right tibia and tarsus showing an inveterate articular fracture of right distal tibial epiphysis with cranial displacement, previously treated with an intramedullary pin (see arrows). The pin does not engage the proximal portion of the tibia and runs for almost its entire length in the caudal portion of the leg. nerve block was carried out with $0.2 \mathrm{ml} / \mathrm{kg}$ of lidocaine (Xylocaine 2\%; Zidus Cadila, India). The affected limb and the contralateral foreleg were clipped, and a $4 \%$ chlorhexidine gluconate detergent was used for the preliminary preparation of the surgical sites, with a minimum contact time of 5 minutes. The patient was positioned in right lateral recumbency, with the affected limb toward the operating table and the contralateral limb fixed in the abduction position. Cefazolin (Cefazoline Teva; Teva s.r.l., Italy) was administered IV (22 mg/kg) approximately 30 minutes before the skin incision. Under complete aseptic precautions, the craniomedial approach was carried out to expose the tarsal joint. A skin incision, the same length as the plate, was made on the caudomedial aspect of the distal tibia and the craniomedial aspect of the tarsus and proximal metatarsus. The intramedullary pin was removed and sent to the laboratory for bacteriological examination. The tarsocrural, intertarsal, and tarsometatarsal joints were exposed, and the articular cartilage was removed with a high-speed burr. The autologous bone graft was collected from the cancellous bone of the contralateral humerus and was subsequently inserted into the joint spaces. After joint reduction, pantarsal arthrodesis was performed. A 1.2-mm Kirschner wire was driven through the calcaneus into the tibia, and a Compact UniLock 2.0 mandible locking plate (DePuy Synthes, Oberdorf, Switzerland) of 1.5-mm thickness was contoured to achieve the functional angle of tarsus extension and was positioned on the medial surface of the tarsus (Fig. 2). The plate was

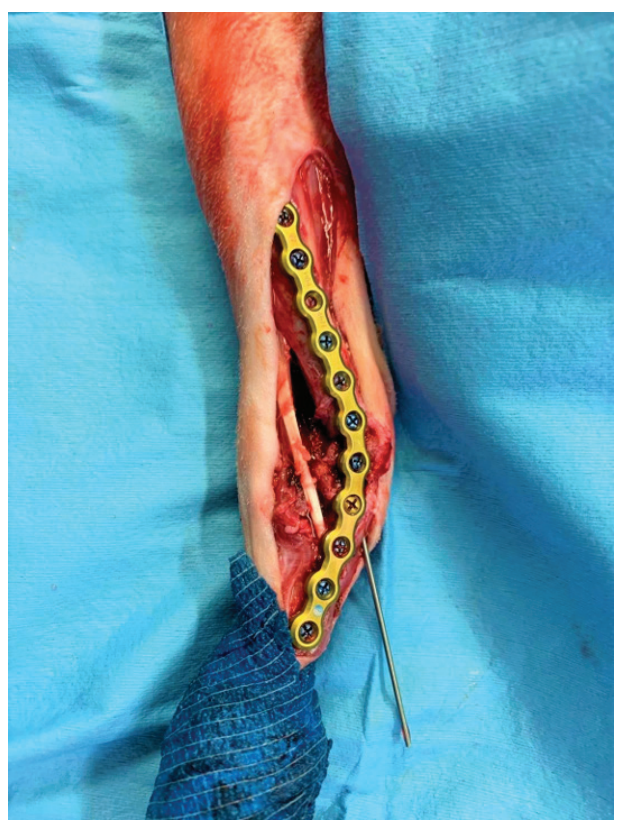

Fig. 2. Intra-operative photograph showing the application of the Compact UniLock 2.0 mandible locking plate on the medial surface of the tarsus. 
fixed with ten $2.0 \mathrm{~mm}$ locking screws and a cortical screw. The soft tissues were sutured by planes using a USP $3 / 0$ absorbable monofilament thread. No tension at the suture lines was observed. The surgery lasted for 63 minutes. At the end of the surgery, an X-ray examination (Fig. 3) showed a good apposition of the fracture, with a slight internal rotation of the calcaneus; the apparatus seemed adequate, with a total plate screw density (PSD) of 0.85 . The joint was fixed at $137^{\circ}$ in extension. After radiographic examination, a soft padded bandage was applied for 14 days, which was renewed weekly. As needed, $0.3 \mathrm{mg} / \mathrm{kg}$ of methadone (Semfortan $10 \mathrm{mg} / \mathrm{ml}$; Dechra, Italy) was administered IM for 24 hours postoperatively. Anti-inflammatory therapy (meloxicam $0.05 \mathrm{mg} / \mathrm{kg}$; Metacam $40 \mathrm{mg} /$ $\mathrm{ml}$; Boehringer Ingelheim Vetmedica, Germany) and ranitidine $(2 \mathrm{mg} / \mathrm{kg}$; Zantadine $30 \mathrm{mg} / \mathrm{ml}$; Ceva s.p.a., Italy) IV were administered on an inpatient basis. The first orthopedic evaluation, the first day after surgery, showed an improvement in gait (grade $2 / 5$ of lameness on walk). The cat was discharged from the hospital after 3 days with a prescription of anti-inflammatory and analgesic therapy. The head of the cat shelter was instructed to subject the cat to cage rest for 30 days. At the first follow-up, 7 days after surgery, the soft padded bandage was renewed, and the cat showed a steady improvement in gait. Fourteen days after surgery, the soft bandage was removed; the orthopedic examination showed mild improvement in gait, although seconddegree lameness and an extended tarsal and flexed stifle gait persisted because of the arthrodesis procedure. No swelling or dehiscence at the surgical site or skin tension under the plate was observed; the suture was removed, and the cat was discharged with light exercise on a larger cage recommendation for the remaining 15 days. One month after surgery, at the orthopedic

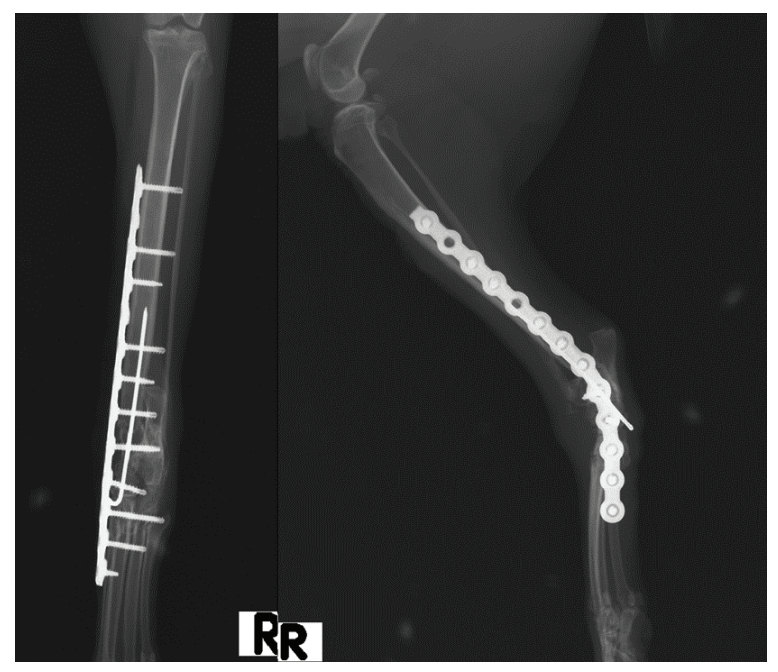

Fig. 3. Craniocaudal and mediolateral postoperative radiographs after pantarsal arthrodesis showing good alignment of the tibia, tarsus, and metatarsus. evaluation, the head of the cat shelter reported a slight worsening of lameness after a sharp jump had occurred 7 days prior. The patient showed good limb function, with a slight worsening of gait during the trot, and the radiographic evaluation showed good bone activity, with $50 \%-66 \%$ of the joint fusion and fracture healed, although a metatarsal stress fracture was revealed (Fig. 4). Four months after surgery, radiographs revealed stable implants and an almost complete fusion of the joints (Fig. 5). The surgical wound site, 30 and 120 days after surgery, healed very well, with an efficient aesthetic result of the limb, even with a slight loss of skin elasticity, which occurs naturally after surgery. The patient never showed discomfort at the implant site, even though the plate was very superficial and palpable under the thin skin (Fig. 6). Moderate free activity with gradual introduction to freedom in the feline colony was prescribed.

\section{Discussion}

The particular condition of our patient, a young stray cat (therefore, wild and without an owner), made pantarsal arthrodesis even more challenging. The pantarsal arthrodesis treatment in this clinical case should, more than ever, cause minimal discomfort to the patient, obtain immediate stability of the implant to avoid bandaging the limb for several weeks, and minimize the impact of any distractions of the patient's holder, who had to take care of several cats inside the shelter. Furthermore, if possible, the implant should be long-lasting and cause as few implant reactions as possible if, after healing, the cat cannot be recaptured to remove the implant. The medial application of a Compact UniLock system achieved all these objectives and showed some advantages over currently available systems.

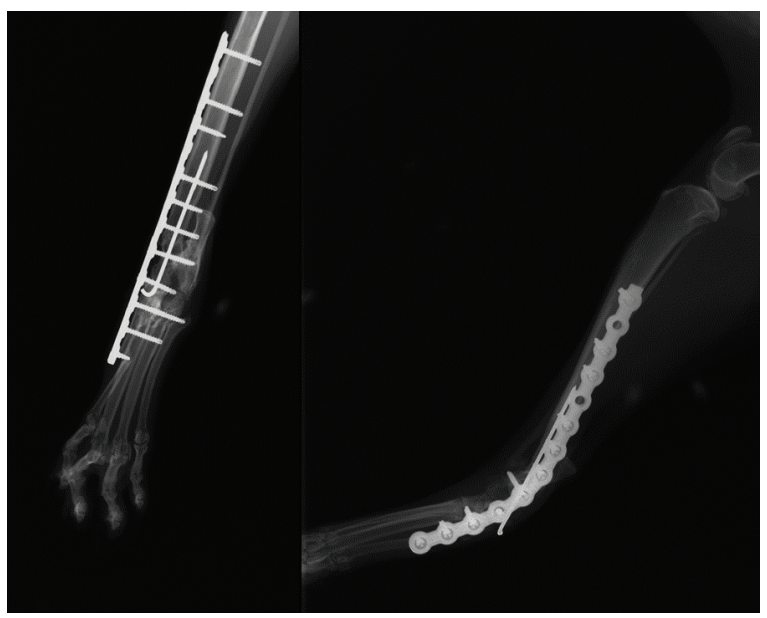

Fig. 4. Radiographic follow-up examination 4 weeks after pantarsal arthrodesis revealing a near fusion of the arthrodesis and a fracture of the second metatarsus. 


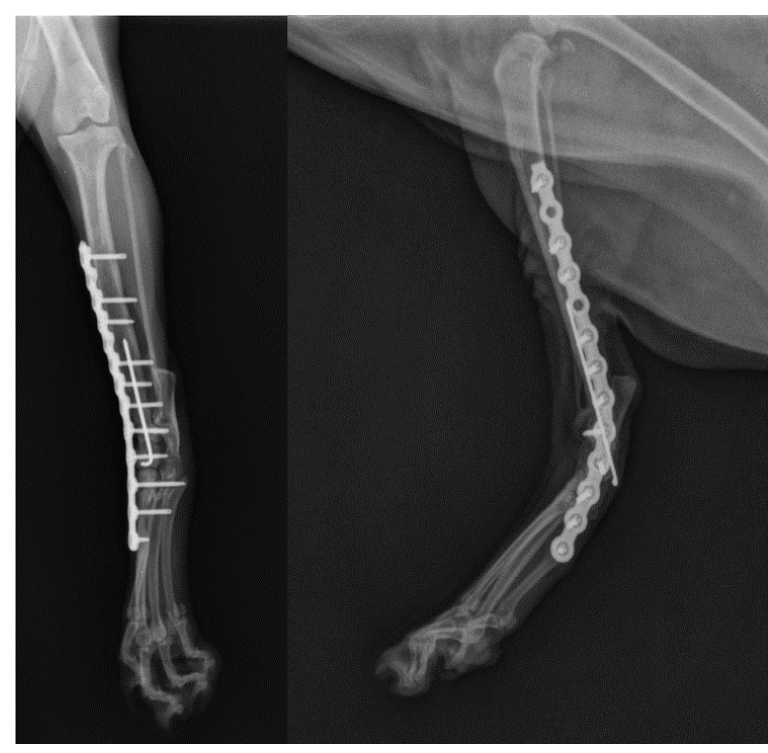

Fig. 5. Radiographic follow-up examination 16 weeks after pantarsal arthrodesis revealing an almost complete fusion of the arthrodesis.

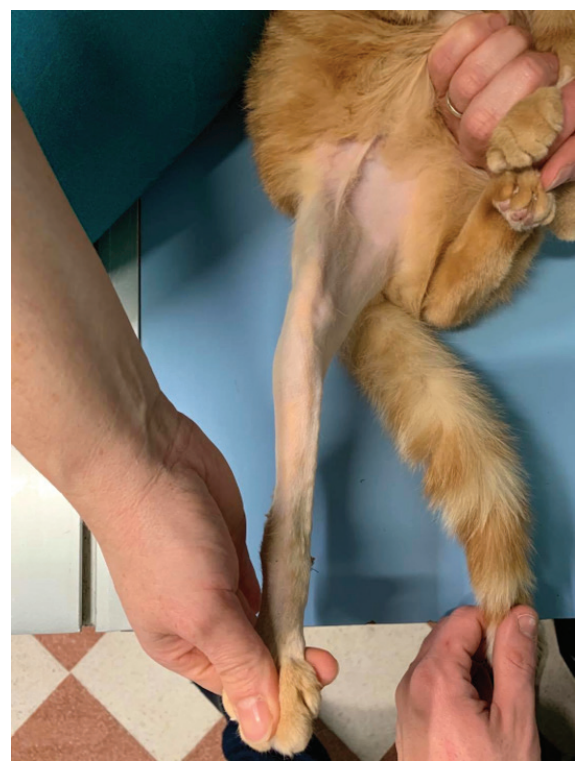

Fig. 6. Photograph after 4 weeks of postoperative recovery showing complete surgical wound healing without skin and soft tissue complications and correct limb alignment.

The tarsus in cats has very small bone segments; therefore, arthrodesis requires a precise application of the plate. The particularly favorable characteristic of the Compact UniLock plate is its easy contouring during surgery, allowing one to apply it precisely to the tarsus shape of our patient, minimizing the risk of incorrectly positioning the screws.
Because of the anatomy of our patient, we were able to insert a small pin and screws perpendicular to the plate and ensure that they were locked properly. The length and thickness of the plate were chosen by the surgeon during operational planning. The ductile feature of the implant prompted the authors to choose a rigid configuration of the apparatus with a plate span ratio 10 times the fracture line and a PSD of 0.85 , even higher than the recommended ratio for the LCP Synthes system (Théoret and Moens, 2007). This fixation system, in addition to the insertion of a small centro-medullary pin, granted high stability and strength to the implant (Gautier and Sommer, 2003; Keller et al., 2005).

As stated by Keller et al. (2005), an advantage of the Compact UniLock system is that the screws create only small protrusions when locked to the plate, permitting and facilitating their placement near tendinous and other soft tissue structures. The possibility of using a very thin locking implant (1.5-mm thickness) has minimized the risk of skin tension and consequent possible plantar necrosis while keeping the configuration safe and stable (Gellrich et al., 2004).

Although the literature suggests applying an external coaptation for 6 weeks after the pantarsal arthrodesis (Mckee, 1994; Harasen, 2002; Streubel et al., 2011), thanks to the stability and stiffness of this locking implant, a bandage needed to be applied for only 2 weeks, resulting in a reduction of post-operative rehabilitation time and complications associated with its use as well as the additional cost and time commitment for its management (Anesi et al., 2019). The very thin locking plate reduced suture skin tension, achieving excellent wound healing and probably a reduction in the risk of serious complications affecting soft tissues described with the use of other traditional or locking medial plate systems (Decamp et al., 1993; Roch et al., 2008; Anesi et al., 2019).

As the patient was a stray cat, we did not know whether we could remove the implant after bone healing. The titanium nature of the adopted system allowed, as reported in the literature, greater biocompatibility compared with stainless steel plates, as evidenced by the rate of infection. Standard implant-quality titanium has a micro-rough surface, which is produced as a result of the selective dissolution of the material at grain boundaries, which produces the characteristic 3D-surface morphology of this material. Consequently, standard titanium supports direct osseointegration, which is highly desirable for long-term/permanent implants, to allow for maximum stability. Even in human medicine, within the last 15 years, electropolished stainless steel implants have been gradually replaced by titanium (Deepak and Manjula, 2011). The driving forces behind this change are related primarily to the improved biocompatibility of the metal and its surface oxides (including extremely fast regeneration), 
superior corrosion resistance, lower stiffness, lower density, and enhanced diagnostic imaging associated with titanium (Hayes and Richard, 2010; Deepak and Manjula, 2011).

Plate loosening and metatarsal bone fractures are reported in the literature as complications of arthrodesis in cats (Calvo et al., 2009). In our case, the fracture of the second metatarsus detected 4 weeks after surgery was due to an accidental traumatic jump of the patient reported from the anamnestic data. The cause of the fracture was linked to the single metatarsal engaged by the last screw. Since the weight of the locking plate is unloaded on the margins of the implant during walking, all the forces are discharged on the second metatarsus, which caused the fracture.

The limitations of this case are represented by the shortterm radiographic and clinical outcomes through joint fusion, the small number of cases (single patient), and the relatively high implant costs compared with other systems. To the authors' knowledge, this is the first case of medial pantarsal arthrodesis with a Compact 2.0 UniLock implant. Future studies could evaluate the long-term outcomes of an increased caseload of cats that undergo pantarsal arthrodesis with this system. In conclusion, pantarsal arthrodesis performed with a Compact UniLock 2.0 plate on the medial aspect of the feline tarsal joint could represent a valid treatment in cases of severe tibiotarsal damage. The titanium implant in association with the intramedullary pin was safe and stable and allowed the complete fusion of the joint and the good functional return of the affected limb. Implant stability has reduced the coaptation time with bandages, decreasing postoperative discomfort in our feline patient. Therefore, the small thickness of the plate and its locking nature, which allows less contact with the bone and no prominence of the screw head from the hole, could reduce vascular damage and skin tension, minimizing the risk of dehiscence and plantar necrosis. Further studies would be needed to evaluate many cases.

\section{Conflicts of interest}

The authors declare no conflicts of interest.

\section{Authors' contributions}

Luca Pennasilico contributed to the study execution, data collection, and writing of the first draft of the manuscript. Riccardo Botto contributed to the study execution, data collection, and critical revision of the manuscript. Caterina Di Bella aided with the data collection, analysis and interpretation of the data, and study design. Angela Palumbo Piccionello aided with the study design, analysis and interpretation of the data, writing of the first draft of the manuscript, and critical revision of the manuscript. All the authors read and approved the final version of the manuscript.

\section{References}

Anesi, S., Clarke, S., Gemmill, T., Oxley, B., Pink, J., Smith, K. and Rutherford, S. 2019. Long-term outcomes after pantarsal arthrodesis with medial plate fixation without external coaptation in 30 dogs. Vet. Surg. 49(3), 502-511.

Calvo, I., Farrell, M., Chase, D., Aisa, J., Rayward, R. and Carmichael, S. 2009. Carpal arthrodesis in cats. Long-term functional outcome. Vet. Comp. Orthop. Traumatol. 22(06), 498-504.

Decamp, C.E., Martinez, S.A. and Johnston, S.A. 1993. Pantarsal arthrodesis in dogs and a cat: 11 cases (19831991). J. Am. Vet. Med. Assoc. 203, 1705-1707.

Deepak, S. and Manjula, S. 2011. Comparison of titanium bone plates and screws vs. stainless steel bone plates and screws in the management of mandibular fractures: a long term clinical study. Int. J. Clin. Dent. Sci. 3(6), 194-199.

Doverspike, M. and Vasseur, P.B. 1991. Clinical findings and complications after talocrural arthrodesis in dogs: experience with six cases. J. Am. Anim. Hosp. Assoc. 27, 553-559.

Dyce, J., Whitelock, R.G., Robinson, K.V., Forsythe, F. and Houlton, J.E.F. 1998. Arthrodesis of the tarsometatarsal joint using a laterally applied plate in 10 dogs. J. Small Anim. Pract. 39(1), 19-22.

Fitzpatrick, N., Sajik, D. and Farrell, M. 2013. Feline pantarsal arthrodesis using pre-contoured dorsal plates applied according to the principles of percutaneous plate arthrodesis. Vet. Comp. Orthop. Traumatol. 26(5), 399-407.

Gautier, E. and Sommer, C. 2003. Guidelines for the clinical application of the LCP. Injury 34, B63-B76.

Gellrich, N.C., Suarez-Cunqueiro, M.M., OteroCepeda, X.L., Schön, R., Schmelzeisen, R. and Gutwald, R. 2004. Comparative study of locking plates in mandibular reconstruction after ablative tumor surgery: THORP versus UniLOCK system. J. Oral Maxillofacial Surg. 62(2), 186-193.

Gorse, M.J., Earley, T.D. and Aron, D.N. 1991. Tarsocrural arthrodesis: long-term functional results. J. Am. Anim. Hosp. Assoc. 27(2), 231-235.

Guillou, R.P., Frank, J.D., Sinnott, M.T., Meyer, E.G., Haut, R.C. and Déjardin, L.M. 2008. In vitro mechanical evaluation of medial plating for pantarsal arthrodesis in dogs. Am. J. Vet. Res. 69(11), 1406-1412.

Harasen, G. 2002. Arthrodesis - Part II: The tarsus. Can. Vet. J. 43(10), 806.

Hayes, J.S. and Richards, R.G. 2010. The use of titanium and stainless steel in fracture fixation. Expert Rev. Med. Devices 7(6), 843-853.

Jaeger, G.H., Marcellin-Little, D.J., DePuy, V. and Lascelles, B.D.X. 2007. Validity of goniometric joint measurements in cats. Am. J. Vet. Res. 68(8), 822-826. 
Keller, M.A., Voss, K. and Montavon, P.M. 2005. The ComPact UniLock 2.0/2.4 system and its clinical application in small animal orthopedics. Vet. Comp. Orthop. Traumatol. 18(2), 83-93.

Klause, S.E., Piermattei, D.L. and Schwarz, P.D. 1989. Tarso-crural arthrodesis: complications and recommendations. Vet. Comp. Orthop. Traumatol. 2(3), 119-124.

McKee, W.M. 1994. Intractably painful joints. In: Manual of small animal arthrology. Eds., Houlton, J.E.F. and Collinson, R.W. Cheltenham, UK: British Small Animal Veterinary Association, pp: 115-134.

MecKe, W.M., May, C., Macias, C. and Lapish, J.P. 2004. Pantarsal arthrodesis with a customised medial or lateral bone plate in 13 dogs. Vet. Rec. 154(6), 165-170.

Roch, S.P., Clements, D.N., Mitchell, R.A.S., Downes, C., Gemmill, T.J., Macias, C. and McKee, W.M. 2008. Complications following tarsal arthrodesis using bone plate fixation in dogs. J. Small Anim. Pract. 49(3), 117-126.

Şirin, Ö.Ş., Şirin, Y.S. and Yiğitarslan, K. 2018. UniLOCK implants for stabilization of long bone fractures: experiences in ten clinical cases. Ankara Univ. Vet. Fak. Derg. 65(2), 179-185.
Spencer, A.J. and Tobias, M.K. 2018. Veterinary surgery small animals, 2nd ed. Philadelphia, PA: Saunders.

Streubel, R., Makara, M. and Guerrero, T. 2011. Medial radio-carpal arthrodesis in three cats with a $2.0 \mathrm{~mm}$ locking maxillofacial plate system. Vet. Comp. Orthop. Traumatol. 24(4), 294-298.

Sumner-Smith, G. and Kuzma, A. 1989. A technique for arthrodesis of the canine tarsocrural joint. J. Small Anim. Pract. 30(2), 65-67.

Théoret, M.C. and Moens, N.M. 2007. The use of veterinary cuttable plates for carpal and tarsal arthrodesis in small dogs and cats. Can. Vet. J. 48(2), 165.

Voss, K., Keller, M. and Montavon, P.M. 2004. Internal splinting of dorsal intertarsal and tarsometatarsal instabilities in dogs and cats with the ComPact UniLock 2.0/2.4 ${ }^{\mathrm{TM}}$ System. Vet. Comp. Orthop. Traumatol. 17(3), 125-130.

Wilke, V.L., Robinson, T.M. and Dueland, R.T. 2000. Intertarsal and tarsometatarsal arthrodesis using a plantar approach. Vet. Comp. Orthop. Traumatol. 13(1), 28-33. 